\title{
Are Radiopharmaceuticals Safe? The Case of FDG-18
}

\author{
Ralph Santos-Oliveira*1, ${ }^{\text {, Brandon Fleming }}{ }^{3}$ \\ ${ }^{1}$ Laboratory of Nanoradiopharmaceuticals, University Hospital Clementino Fraga Filho, Federal University of Rio de Janeiro \\ ${ }^{2}$ Nuclear Engineering Institute, Education Coordination
}

${ }^{3}$ Mathematics \& Statics Department, University of Maryland, Baltimore County (UMBC)

\begin{abstract}
Too many false-positive and adverse reactions related to radiopharmaceuticals take place every day in hospitals routine, but many are not reported or even sensed. Information concerning these kind of reactions is not abundant and nuclear medicine staff is usually overwhelmed by this information. As every healthcare intervention carries some risk of harm, clinical decision making needs to be supported by a systematic assessment of the balance of benefit to harm. A systematic review that considers only the favorable outcomes of an intervention, without also assessing the adverse effects and false positives reactions, can mislead by introducing a bias favoring the intervention that in the case o radiopharmaceuticals may to render a important factor related not even to the quality of the drug but even to the quality of the diagnosis. The results suggest a logical framework to make decisions in reviews that incorporate false positive and adverse reactions. Also, was explored situations where a comprehensive investigation of false positive reactions and adverse effects was warranted and suggest strategies to identify practicable and clinically useful outcomes. We concluded that there is the necessity to include and to recognize how strategic choices made in the review process determine what harms are found, and how the findings may affect clinical decisions. Researchers undertaking a systematic review that incorporates false positive reaction and adverse reactions must understand the rationale for the suggested methods and be able to implement them in their review. Beyond a world effort should be made to report as many cases of false positive and adverse reactions with radiopharmaceuticals as possible. Only if this is done a complete picture of false positive reactions with radiopharmaceuticals can be drawn.
\end{abstract}

Keywords: Radiopharmaceuticals; Safety; Nuclear Medicine; Oncology; Radiology

\section{Introduction}

In 1967, Numerof [1] stated that nuclear medicine has grown in recent years from an esoteric field populated by a small group of closely allied health care providers into large multidisciplinary medical teams with a scope of practice that cuts across multiple disciplines. This observation rings true even more so today. There are two requirements necessary in any nuclear medicine procedure. First the a drug (radiopharmaceutical) must be available, and secondly it has to be properly labelled as a tracer. The field of radiopharmacy and radiopharmaceutical has grown substantially since 1924, when Herman Blumgart conducted the first clinical tracer studies by injecting radon and later, solutions of $214-\mathrm{Bi}$, into one arm of a patient and observing the time it took to reach the other arm [2].

According to Mather [3] radiopharmacy is scientifically recognised as an essential sub-speciality of nuclear medicine. Without radiopharmaceuticals, the "food" of nuclear medicine, no radiodiagnostic or radiotherapeutic procedures could be performed, and without progress in radiopharmacy medical speciality, like nuclear medicine, will, ultimately, wither and die. Radiopharmaceuticals serve two complementary roles as a diagnostic tool. The first is as a measure bioavailability: the labeled compound is administered to the patient and some aberrant physiological or biochemical process leads to an abnormal distribution of the compound in the patient. The second role the radiopharmaceutical is as a tracer for particular physiological or biochemical process and the time course of its distribution is used to quantity the biological process $[4,5]$.

The last few decades have seen an immense growth in availability and consumption of medicines. Whilst most consumers derive far more benefit than harm, a proportion of patients experience undesirable side effects or adverse drug reactions (ADR) (from the use of medicines at even the recommended dosage and dosing schedule [6]. Radiopharmaceuticals are no different; however, the consequence of an ADR can be much more severe. Moreover, the interference is directly related to the quality of the exam which may result in a false positive reaction.

Jones [7] has already alerted to the fact that although ADRs can appear as isolated, specific clinical events that may be related to a number of factors in the patient's background and environment, in many situations it may not appear early as a detectable clinical event, but rather remain clinically silent. Again as drugs, radiopharmaceuticals are no different. Other descriptive characteristics of ADRs concern their acuteness or latency relative to drug exposure, their severity, and their frequency or incidence. The latter information, although highly desirable, is usually not well-defined for most cases of ADR, with the exception of the common ones defined in clinical trials. The incidence of rarer effects is known for just a few drugs, as only recently have epidemiological methods and studies been directed towards the study of pharmacovigilance and post-market drug safety.

According to Silberstein and Ryan [8], significant adverse drug reaction should be reported must include the following:

*Corresponding author: Ralph Santos-Oliveira, Nuclear Engineering Institute Rua Hélio de Almeida 75 - Ilha do Fundão, Rio de Janeiro, Brazil-21941906, Tel: +552121733964; Fax: +552121733852; E-mail: roliveira@ien.gov.br

Received November 28, 2011; Accepted January 16, 2012; Published January 20, 2012

Citation: Santos-Oliveira R, Fleming B (2012) Are Radiopharmaceuticals Safe? The Case of FDG-18. Adv Pharmacoepidem Drug Safety 1:102. doi:10.4172/ 2167-1052.1000102

Copyright: (c) 2012 Santos-Oliveira R et al. This is an open-access article distributed under the terms of the Creative Commons Attribution License, which permits unrestricted use, distribution, and reproduction in any medium, provided the original author and source are credited. 
1. Untoward effects whether observed frequently or rarely

2. Untoward effects never before seen following administration of the radiopharmaceutical

3. Only life-threatening or fatal reactions from drugs other than radiopharmaceuticals

4. Reactions unanticipated from unknown pharmacological action of a nonradioactive pharmaceutical 5. Anaphylactoid or allergic reactions.

Nonetheless, despite the potential for severe ADR in patients who are using a radiopharmaceutical, the actual incidence of ADR is rarer when compared to other drugs for therapeutic purposes. Nevertheless the incidence of false positives remain significant [9]. The results of both ADR and false positives are linked to radiopharmaceuticals show just a few incidents, but that may be due to the small number of reported cases. Studies demonstrated that only $10 \%$ of possible reactions are actually reported [10]. Besides, the great majority of these are neither serious/life-threatening nor require active treatment [10].

Estimates of adverse reaction prevalence and false positive reactions are difficult to assess, partly because of physician/doctor ignorance of available reporting schemes. The reasons for not filling out adverse reactions reporting forms are numerous, and may include subjective nature of interpreting an adverse reactions, emotional considerations such as a physician's anxiety about potential liability, or the belief that the reaction is common knowledge $[8,11]$.

\section{Methodology}

As with any systematic review of effectiveness, a clearly focused research question is essential in a review of adverse effects (ADR) and false positive reactions. Relevant questions will be those that are directly aimed at guiding the decisions of clinicians, consumers, researchers and policymakers, in this direction, a protocol should be developed for the systematic review and details of the research question specified, including the types of participants, interventions/exposures, comparisons, outcomes, and study designs to be included. Selection of outcomes and study type requires careful consideration in reviews of false positive reactions and adverse affects and should be made by specialized staff. Systematic reviews which include false positive and adverse reactions are likely to make more than one comparison. In such a case, the early stages of the protocol should specify what the main comparisons will be and whether the review will consider both beneficial and not beneficial reactions, in this case false positive reactions and adverse effects. The resources devoted to studying false positive reactions and adverse effects outcomes should reflect the importance of the treatment in context. For example, if a treatment confers little benefit and is seldom used, its adverse effects or even its false positive reactions may not be worth a detailed evaluation. Some interventions will require an exhaustive analysis of all harmful outcomes, while others may require a less thorough investigation [12].

Usually, when the focus of the research question is purely on safety and or tolerability, the effectiveness of the treatment is already known or defined [13]. However, in some instances reviewers intend to evaluate adverse effects and/or false positive reactions as part of a combined review that also covers beneficial outcomes. Reviews that aim to evaluate benefit and harm together will usually require a more complex study design that can efficiently handle different sets of studies for various outcomes. Using different search strategies and/or eligibility criteria for studies of benefit and harm will generate two or more diverse groups of eligible studies. Unlike reviews of effectiveness, where all beneficial outcomes are likely to be well recognized beforehand, in these types of studies specific adverse effects associated with a therapeutic intervention may or may not be known in advance of the review. It may not be possible to specify in advance which effects will be most relevant to the review. Moreover, the research question about safety and tolerability in a review can be broad or narrow in scope. In general, reviewers who have already identified important safety concerns should carry out a narrow-focused evaluation covering particular aspects of the relevant adverse effects and false positive reactions. In relation to the types of studies no single recommendation is possible here, and any decisions have to be made case by case. The decision on what types of studies to include will depend primarily on the main focus of the research question, balancing the elements of type of adverse effects(s) of interest, rigour in searching, and time and resources available. The systematic evaluation of new or rare adverse effects and even specifically false positive reactions may require the inclusion of other study designs: cohort, case-control, cross-sectional, and even case series. Specific to adverse reactions a recommendation is important: authors planning to use such additional data sources should realize that estimates of the frequencies of adverse effects from published case reports and spontaneous reporting may differ greatly from the results obtained from a meta-analysis of double-blind, randomized controlled trials [14]. A study comparing adverse outcomes from randomized and nonrandomized studies found that the latter often yield lower estimates of absolute risk of harm [15].

The location and the selection of studies by the authors is one of the most important step in a systematic review. The authors need to develop a literature search strategy based on key elements in their research question: population, intervention (plus acceptable comparators), and outcomes. The review question determines the nature of the search strategy. In this study a review of the literature on adverse reaction and false positive reactions with radiopharmaceuticals was carried out. Computerized databases for radiopharmaceuticals were searched, such as MEDLINE, EMBASE, International Pharmaceutical Abstracts and Science Citation Index, published between 1956 and 2007 (April). The searches were supplemented with manual searches of bibliographies of the published articles on major radiopharmacy textbooks, and in the Cochrane Database of Systematic

Two main approaches can be used, each with its own limitations; in the case of a systematic review of adverse effects and false positive reactions related to the FDG-18. They are best combined to maximize sensitivity:

\section{Electronic databases using index terms}

Electronic databases using index terms (also called controlled vocabulary or thesaurus terms): a) Index terms such as MeSH (Medical Subject Headings) in MEDLINE and EMTREE in EMBASE are assigned to records in electronic databases to describe the studies. Subheadings can also be added to index terms to describe specific aspects - for example, side effects of drugs, or complications of surgery. In this study the indexing terms used were: adverse reactions; contraindications, complications, false positive reactions, misdiagnosis, false negative; toxicity and others. b) Indexing terms in MEDLINE and EMBASE: Within a database, studies may be indexed in three different ways: (i) under the name of the intervention together with a subheading to denote that adverse effects and false positive reactions occurred, for example, FDG/adverse effects, PET/complications; (ii) under the adverse event itself, together with the nature of the intervention, for example, Misdiagnosis/and FDG/, arthroplasty/and surgery/; or 
(iii) occasionally only under the adverse event, for example, adverse reaction/chemically-induced. Thus, no single index or subheading search term can be relied on to identify all data on adverse effects, but a combination of index terms and subheadings will help to detect reports of major adverse effects which the indexers have considered significant $[16,17]$.

\section{Electronic databases using free-text terms ('text words')}

Electronic databases using free-text terms ('text words') Terms used by authors in the title and abstract of their studies can be searched on databases of electronic records using free-text terms. Two problems seriously limit the value of free-text searching:

- Authors commonly use a wide range of terms to describe adverse effects, both in a general sense (toxicity, side-effect, adverse-effect) and more specifically (eg, lethargy, tiredness, malaise may all be used synonymously). An author of a review may not know all these terms in advance but should try to include as many relevant synonyms as possible.

- The free-text search does not detect adverse effects or false positive reactions not mentioned in the title or abstract of the study in the electronic record (even though they appear in the full report) $[12,17]$.

A sensitive free-text search should incorporate this potentially wide variety of synonymous terms used to denote data on adverse effects in studies, while also taking into account different conventions in spelling and variations in the endings of terms. So it is necessary to include singular and plural terms. These terms used to describe adverse effects and false positive reactions should then be combined with freetext terms used to describe the intervention of interest. A systematic review with a focus adverse effects and false positive reactions, difficult to conduct for normal "cold" drugs the difficulty is only increase in the case of radiopharmaceuticals. Clearly no single approach will reliably yield all the studies that have data on adverse effects of an intervention. The search, therefore, needs to combine index terms and free-text terms and is likely to take several iterations. In deciding which combination of terms to use, authors will need to balance comprehensiveness (sensitivity) against precision. For example, an electronic search that retrieves 20,000 studies is likely to contain most of the relevant studies, but if only $300(1.5 \%)$ are relevant it is very imprecise and resource intensive [12,18]. Review authors planning an exhaustive search may wish to consider checking other sources of information on adverse effects and false positive reactions, which include spontaneous reporting systems and data from regulatory agencies both done in this study as described above. An important factor that must be considered is that there is often a major trade-off between the comprehensiveness and the quality of the adverse effects data and false positive reactions data included in a systematic review. Including evidence that is likely to be biased, even if no better evidence exists, may lead to biased conclusions. All included data should be critically discussed and rigorously appraised.

One cannot conclude from 'zero events detected' that an intervention does not cause a suspected adverse effect or even a false positive reaction. However, we can use the rule of three (for sample sizes > 30), to estimate the upper limit of the $95 \%$ confidence interval for the probability of the adverse effect [19]. If no events were detected in $n$ trial participants, the upper limit of the $95 \%$ confidence interval for the probability of the adverse event is $3 / \mathrm{n}$. In effect, this gives us a good idea of what the worst-case scenario could be, if no adverse events have occurred so far in our sample. For example, if no adverse effects occur in 300 participants, then any adverse effects associated with the intervention might be as frequent as 1 in 100 , but are unlikely to be more frequent. Note that studies with no events in either arm can be included in a meta-analysis of risk differences, but they cannot be included in a meta-analysis of odds ratios or risk ratios.

It is important to remember that a systematic review is not synonymous with a meta-analysis. In many circumstances adverse effect and false positive information is best summarised in a qualitative or descriptive manner. For instance, data derived from divergent sources cannot be combined because of different study design, different populations or, different data collection methods. It may not be possible to compare benefits and harms directly. In practice this means that adverse effects from case reports, case series, cohorts, and case controls cannot all be pooled together using standard meta-analysis principles. Further, the data from non-randomized studies are more prone to bias, and are often heterogeneous; suspected heterogeneity need to be considered before such studies are combined to generate a summary statistics.

\section{Sarcoid reaction to FDG}

In recent years PET scans with fludeoxyglucose (FDG-18) have been playing an increasingly important role in the evaluation of the response to induction chemotherapy, as well as in the detection of primary tumor and metastatic lesions in several malignancies [20-22]. In one reported case a 66 year-old man was referred to the hospital for investigation of an abnormal shadow measuring $5.2 \mathrm{~cm}$ in diameter in the left upper lung field on a chest X-ray. The patient was eventually diagnosed with lung cancer classified as clinical stage IIIA (T2N2M0) and underwent induction chemotherapy with paclitaxel.

On the other hand, an FDG-PET scan prior to the chemotherapy demonstrated accumulation only in the tumor and not in the mediastinal node. This result led to suspicion that the disease had progressed to N3 lesions. Hence the patient underwent a biopsy of the right supraclavicular lymph node and the mediastinal lymph nodes by mediastinoscopy. All dissected lymph nodes showed sarcoid reactions and no tumour cells were found to be pathological. The patient had no clinical symptom of sarcoids and the chest X-ray did not show streaks and/or spots. The conclusion was that a sarcoid reaction of the mediastinal lymph nodes probably led to the abnormal accumulation of FDG without tumor metastasis. Therefore, in patients with FDGPET positive results, it is necessary to verify lymph node pathologically using mediastoscopy, because this type of adverse reaction (sarcoid reaction) may occur and change the clinical conduction [20].

Another case of sarcoid reaction occurred with a 57-year-old female patient with a history of diffuse large B-cell lymphoma, involving the lungs and spleen. This patient received 8 courses of $\mathrm{CHOP}$ (cyclophosphamide, doxorubicin, vincristine, prednisone) and rituxan therapy. After 4 months she underwent a PET-CT scan that showed increased uptake in the spleen. This patient underwent a splenectomy due to the suspicion of recurrence. The results of the pathology showed noncaseating granulomas consistent with sarcoid-like reaction. These two cases show the importance of the sarcoid-reaction and the importance of a pathological analysis in case of suspicion [23].

\section{False Positive Reactions}

Another case of false positive uptake, described by Iwasaki et al. [24] with a 57-year-old woman, demonstrated that fused FDG-PET and PET/CT images performed to analyze pulmonary suture abscess can be confused with lung cancer, because the abscess, as an inflammatory 
process, increase the uptake of FDG by the lesion. During the analysis, the scan data can be misunderstood as a lung cancer. A report made by Aide et al. [25] described a false-positive uptake of FDG in three patients related to foreign body reaction. According to the authors remote mesh prostheses can induce FDG uptake because of persistent foreign body reaction. Consequently, in each scan an unexpected pelvic focus was noticed on FDG PET, since that is where the prostheses were. The authors confirmed that the medical history of patients should be carefully reviewed to avoid false-positive results.

Hurwitz [26] described a 54-year-old woman with a treated carcinoma of the breast. There was spontaneous rupture of an implant placed previously as part of breast reconstruction. An FDG-PET scan was carried out and disclosed intense uptake in these nodes. Lymph nodes biopsy, however, demonstrated benign inflammatory reaction and no recurrence of malignancy. A false-positive PET scan may occur when FDG is taken up in a ruptured breast implant and data should be carefully analyzed in these cases.

Other case of false-positive was described by Modi et al. [27] with a 73-year-old man that received Teflon to treat a vocal cord paresis and showed abnormal uptake of FDG during a PET scan. According to the authors the false-positive response was due a foreign body reaction (as described above) related to Teflon.

An important case of false-positive reaction is related to the hip arthroplasty. A retrospective and prospective study conducted by Zhuang et al. [28] with nine patients and 710 patients respectively, concluded that, following hip arthroplasty, non-specifically increased FDG uptake around the head or neck of the prosthesis persists for many years, even in patients without any observable complications. Therefore, to minimize the number of false-positive results with PET studies, caution should be exercised when interpreting FDG uptake around the head or neck portion of prostheses.

Recently another case o false positive reaction was published. A 70-year-old woman receiving long-term corticosteroids therapy for sarcoidosis presented with hip pain. She underwent wholebody technetium $99 \mathrm{~m}$ methylene diphosphonate bone scintigraphy to exclude vascular necrosis of the femoral head. Although there was no suggestion of vascular necrosis, multiple areas of increased activity were present in the axial and proximal appendicular skeleton. These findings suggested multiple skeletal metastases. A computed tomography scan of her chest showed bilateral pulmonary opacities and an infiltrating process involving the mediastinum and both hila. A computed tomography scan of her abdomen identified splenomegaly and lymphadenopathy. The differential diagnosis included smallcell lung cancer, sarcoma, lymphoma and infection. The patient had received a diagnosis of sarcoidosis 5 years earlier when she presented with lymphadenopathy and night sweats. The initial workup included a computed tomography scan that showed lymphadenopathy and an axillary lymph-node biopsy that revealed noncaseating granulomata. The radiologist reviewing the current computed tomography scan did not receive this background information and suspected a malignant process. The patient's respirologist subsequently performed bonemarrow biopsies, which demonstrated noncaseating granulomata consistent with skeletal sarcoidosis. The patient's condition has been stable, and a repeat bone scan after 2 years of follow-up showed no change. This case demonstrates a rare but important manifestation of sarcoidosis mimicking skeletal metastases on bone scintigraphy. It underscores the importance of providing complete clinical information to the imaging service to facilitate accurate diagnostic interpretation [29].

\section{Conclusion}

The literature review on this topic reveals only a few studies were made that address the issue of safety (ADR) and effectiveness (false positives) with radiopharmaceuticals, despite their increase use; this disproportion between increase use and low reporting would suggest that there is a tremendous amount of possible underreporting of ADR and false positives with radiopharmaceuticals usage. As a sizable proportion of these cases are suspected have hidden false positives, this is a clear justification (if indeed any is needed) for further investigations in this area of research concerning ADR and false positives of radiopharmaceutical products.

\section{References}

1. Numerof $P$ (1967) Radiopharmaceutical development in nuclear medicine Lahey Clinic Foundation Bulletin 16: 321-326.

2. Early PJ (1995) Use of diagnostic radionuclides in medicine. Health Phys 69 649-661.

3. Mather SJ (2001) Innovation in radiopharmacy: progress and constraints? Eur J Nucl Med 28: 405-407.

4. Tewson TJ, Krohn KA (1998) PET radiopharmaceuticals: state-of-the-art and future prospects. Semin Nucl Med 28: 221-234.

5. Baranowska-Kortylewicz J (2007) Radioactive drugs in drug developmen research: quality assurance issues. Mini Rev Med Chem 7: 231-244.

6. Wiffen P, Gill M, Edwards J, Moore A (2002) Adverse drug reactions in hospita patients - a systematic review of the prospective and retrospective studies. Bandolier Extra.

7. Jones JK (1982) Adverse drug reactions in the community health setting: approaches to recognizing, counseling, and reporting. Fam Community Health 5: 58-67.

8. Hesslewood SR, Keeling DH (1997) Frequency of adverse reactions to radiopharmaceuticals in Europe. Eur $\mathrm{J}$ Nucl Med 24: 1179-1182.

9. Keeling DH (1994) Adverse reactions and untoward events associated with the use of radiopharmaceuticals. Textbook of radio pharmacy theory and practice. Gordon and Breanch Sciece Publishers 285-295.

10. Silberstein EB, Ryan J (1996) Prevalence of adverse reactions in nuclear medicine. Pharmacopeia Committee of the Society of Nuclear Medicine. J Nucl Med 37: 185-192.

11. Silberstein EB (1998) Prevalence of adverse reactions to positron emitting radiopharmaceuticals in nuclear medicine. Pharmacopeia Committee of the Society of Nuclear Medicine. J Nucl Med 39: 2190-2192.

12. Loke YK, Price D, Herxheimer A, Cochrane Adverse Effects Methods Group (2007) Systematic reviews of adverse effects: framework for a structured approach. BMC Med Res Methodol 7: 32.

13. Jefferson T, Price D, Demicheli V, Bianco E, European Research Program for Improved Vaccine Safety Surveillance (EUSAFEVAC) Project (2003) Unintended events following immunization with MMR: a systematic review. Vaccine 21: 3954-3960.

14. Loke YK, Derry S, Aronson JK (2004) A comparison of three different sources of data in assessing the frequencies of adverse reactions to aminodarone. $\mathrm{Br} J$ Clin Pharmacol 57: 616-621.

15. Papanikolaou PN, Christidi GD, loannidis JP (2006) Comparison of evidence on harms of medical interventions in randomized and nonrandomized studies. CMAJ 174: 635-641.

16. Derry S, Loke YK, Aronson JK (2001) Incomplete evidence: the inadequacy of databases in tracing published adverse drug reaction in clinical trials. BMC Med Res Methodol 1: 7.

17. Golder S, Mclntosh HM, Duffy S, Glanville J, Centre for Reviews and Dissemination and UK Cochrane Centre Search Filters Design Group (2006) Developing efficient search strategies to identify reports of adverse effects in MEDLINE and EMBASE. Health Info Libr J 23: 3-12.

18. McIntosh HM, Woolacott NF, Bagnall AM (2004) Assessing harmful effects in systematic reviews. BMC Med Res Methodol 4: 19. 
Citation: Santos-Oliveira R, Fleming B (2012) Are Radiopharmaceuticals Safe? The Case of FDG-18. Adv Pharmacoepidem Drug Safety 1:102. doi:10.4172/2167-1052.1000102

Page 5 of 5

19. Thomson LE, Allman KC (2001) Erythema multiforme reaction to sestamibi. J Nucl Med 42: 534

20. Maeda J, Ohta M, Hirabayashi H, Matsuda H (2005) False positive accumulation in 18-F fludeoxyglucose positron emission tomography scan due to sarcoid reaction following induction chemotherapy for lung cancer. Jpn J Thorac Cardiovasc Surg 53: 196-198.

21. Weber WA, Petersen V, Schmidt B, Tyndale-Hines L, Link T, et al. (2003) Positron emission tomography in non-small-cell lung cancer: prediction of response to chemotherapy by quantitative assessment of glucose use. J Clin Oncol 21: 2651-2657

22. Lindsay MJ, Siegel BA, Tunis SR, Hillner BE, Shields AF, et al. (2007) The National Oncologic PET Registry: Expanded Medicare Coverage for PET under coverage with evidence development. AJR Am J Roentgenol 188: 1109-1113.

23. Reilly TB, Schuster DM, Starsiak MD, Kost CB, Halkar RK (2007) Sarcoid-like reaction in the spleen following chemotherapy for non-Hodgkin's lymphoma. Clin Nucl Med 32: 569-571.

24. Iwasaki T, Nakagawa K, Katsura H, Nakane S, Kawahara K, et al. (2006)
Pulmonary suture abscess with false-positive 18F-fluordeoxyglucose positron emission scan mimicking lung cancer recurrence. Jpn J Thorac Cardiovasc Surg 54: 351-355.

25. Aide N, Deux JF, Peretti I, Mabille L, Mandet J, et al. (2005) Persistent foreign body reaction around inguinal mesh prostheses: a potential pitfall of FDG pet AJR Am J Roentgenol 184: 1172-1177.

26. Hurwitz R (2003) F-18 FDG positron emission tomographic imaging in a case of ruptured breast implant: inflammation or recurrent tumor? Clin Nucl Med 28 755-756.

27. Modi D, Fulham MJ, Mohamed A, Havas TE (2005) Markedly increased FDG uptake in a vocal cord after medialization with teflon: pet/ct findings. Clin Nucl Med 30: 45-47.

28. Zhuang H, Chacko TK, Hickeson M, Stevenson K, Feng Q, et al. (2002) Persistent non-specific FDG uptake on pet imaging following hip arthroplasty Eur J Nucl Med Mol Imaging 29: 1328-1333.

29. Nijjar SS, Leslie WD (2008) A case of skeletal sarcoidisis imitating skeleta metastases on bone scintigraphy. CMAJ 178: 153-154. 